\title{
Time From the Beginning of the Right Ventricle Isovolumetric Contraction to the Peak of the S Wave: A New TDI Indicator for the Non-Invasive Estimation of Pulmonary Hypertension
}

\author{
Mozhgan Parsaee, ${ }^{1}$ Fereshteh Ghaderi, ${ }^{2,}{ }^{*}$ Azin Alizadehasl, ${ }^{1}$ and Hooman Bakhshandeh ${ }^{3}$ \\ ${ }^{1}$ Echocardiography Research Center, Rajaie Cardiovascular Medical and Research Center, Iran University of Medical Sciences, Tehran, IR Iran \\ ${ }^{2}$ Department of Cardiovascular Medicine, Echocardiography laboratory, Atherosclerosis Prevention Research Center, School of Medicine, Mashhad University of Medical \\ Sciences, Mashhad, IR Iran \\ ${ }^{3}$ Rajaie Cardiovascular Medical and Research Center, Iran University of Medical Sciences, Tehran, IR Iran \\ "Corresponding author: Fereshteh Ghaderi, Department of Cardiovascular Medicine, Echocardiography laboratory, Atherosclerosis Prevention Research Center, School of \\ Medicine, Mashhad University of Medical Sciences, Mashhad, IR Iran. Tel: +98-9137913316, Fax: +98-5138544504, E-mail: drghaderif@yahoo.com
}

Received 2015 January 09; Revised 2015 October 21; Accepted 2015 November 03.

\begin{abstract}
Background: Echocardiography is a key screening tool in the diagnostic algorithm of pulmonary hypertension (PH). In addition, tissue doppler imaging (TDI) is a promising method for the noninvasive estimation of pulmonary artery pressure (PAP).

Objectives: The aim of this study was to validate the accuracy of measuring the time from the beginning of the right ventricular isovolumetric contraction time (RV IVRT) to the peak of the $S$ wave in the TDI of the base of the RV free wall (time to peak or TTP), as an indicator for the non-invasive estimation of pulmonary hypertension.

Patients and Methods: In this diagnostic test study, 60 consecutive patients referred for right heart catheterization (RHC) were enrolled. A pulse-wave TDI was performed before the cardiac catheterization, with a mean interval of 1 hour between the two measurements. The TDI variables, such as the RV IVRT, myocardial performance index (MPI), and the new "time to peak" parameter, were measured at the lateral basal RV free wall. The patients were divided into two sub-groups according to the RHC findings: no$\mathrm{PH}$ (mean PAP $<25 \mathrm{mmHg}$ ) and PH (mean PAP $\geq 25 \mathrm{mmHg}$ ) groups. Then, we calculated the specificity and sensitivity of the TDI parameters (including the TTP) for the diagnosis of $\mathrm{PH}$.

Results: In our study, the TTP showed a significant inverse relationship with the PAP. Based on our results, a TTP of less than $127 \mathrm{~ms}$ could be used to predict $\mathrm{PH}$, with a sensitivity and specificity of about 70\% (AUC $=0.746 \pm 0.064$ ).

Conclusions: Based on the results of this study, we suggest the use of a novel "time from the beginning of isovolumetric contraction to the peak of the $S$ wave" (TTP) parameter in the TDI of the base of the RV free wall to predict PH with acceptable accuracy in comparison with RHC.
\end{abstract}

Keywords: Tissue Doppler Imaging, Right Heart Catheterization, Pulmonary Artery Pressure, Hypertension, Pulmonary

\section{Background}

Pulmonary artery pressure (PAP) is an important hemodynamic variable which reflects the different volumes and pressures in the left and right sides of the heart (1-4). The standard method for evaluating the PAP is invasive right heart catheterization (RHC), and the pulmonary hypertension $(\mathrm{PH})$ is defined as the mean PAP of $\geq 25$ $\mathrm{mmHg}$ at rest, as assessed by $\mathrm{RHC}(5,6)$.

Echocardiography provides a bedside, simple, and noninvasive way for estimating the $\operatorname{PAP}(3,5)$. The principal approach for estimating the systolic PAP (SPAP) involves the measurement of the tricuspid regurgitation (TR) velocity, as calculated by the Bernoulli Equation. The The spectral Doppler profile of the TR is too weak in approximately 10 to $25 \%$ of those patients with clinical signs of elevated right heart pressure $(3,7,8)$. Therefore, an alternative and effective method for estimating the PAP would be valuable in these patients.

Current guidelines recommend that a detailed echocardiographic assessment be performed in all patients with suspected PH. Several novel echocardiographic indices that allow an evaluation of the PAP have been introduced by tissue doppler imaging (TDI). Therefore, the present study aimed to validate the pulse-wave TDI parameters of the lateral tricuspid annulus, including the right ventricular isovolumetric contraction time (RV IVRT), RV myocardial performance index (RV MPI), and T peak against the invasively measured PAP.

\section{Objectives}

Considering the fact that there is insufficient TR Doppler signal to determine the systolic PAP in a signifi- 
cant number of patients using conventional echocardiography, we decided to evaluate the relationship between the new echocardiographic indexes using the pulse-wave TDI method and a novel parameter: time from the beginning of the isovolumetric contraction to the peak of the $S$ wave in TDI (time to peak or TTP) to estimate the mean PAP measured by RHC to predict PH.

\section{Patients and Methods}

\subsection{Study Population}

This was a cross sectional study to assess the accuracy of the TTP, when compared to RHC, as the standard for the diagnosis of PH. This research was conducted from February of 2013 until March of 2014 at the Rajaie cardiovascular medical and research center, a tertiary care center for cardiovascular patients in Tehran, Iran. In this period, 60 patients who were candidates for RHC were considered to be eligible for the study, and the research protocol was approved by the institutional research committee of our center. To control the confounding variables, only those patients with a normal sinus rhythm at the time of the examination were enrolled. Patients with an age $<18$ years, a right or left bundle branch block (QRS $\geq 120 \mathrm{~ms}$ ), a history of coronary artery disease, complex congenital heart disease or tricuspid and pulmonary stenosis, and those with a history of open heart surgery were excluded from the study.

\subsection{Cardiac Catheterization}

Following local anesthesia, RHC was performed using a multipurpose catheter inserted through the right femoral vein, and the mean PAP was determined at the endexpiration time. The patient population was divided into two groups based on the RHC results: no-PH (mean PAP < $25 \mathrm{mmHg}$ ) and $\mathrm{PH}$ (mean PAP $\geq 25 \mathrm{mmHg}$ ) groups.

\subsection{Echocardiography and Tissue Doppler Imaging}

An echocardiographic study was performed before cardiac catheterization, with a mean interval of 1 hour between the two measurements in a fasting and clinically stable status. The patients underwent a routine transthoracic echocardiographic examination in the left lateral decubitus position, using commercially available equipment (Vivid 3, GE, USA) with a 3-MHz transducer. A pulse-wave TDI was performed in the apical 4-chamber view using a sample volume size $(6 \mathrm{~mm}$ ) adequate to cover the basal RV longitudinal excursion. The RV IVRT was measured as the time between the end of the systolic wave (Sm) and the beginning of the diastolic wave (Em) (Figure 1). The adjusted RV IVRT was calculated as the RV IVRT $/ \sqrt{ }$ RR.

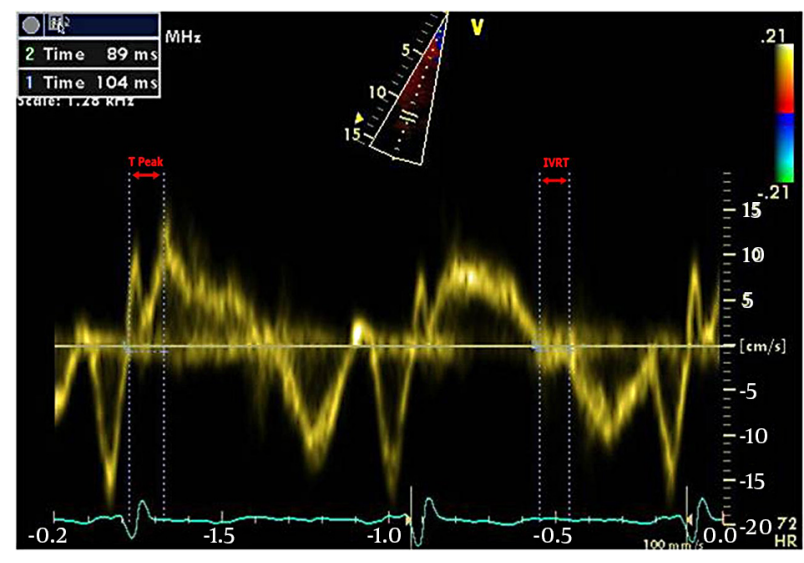

Figure 1. TDI From the Beginning of the Isovolumetric Contraction to the Peak o the Systolic Wave (TTP), Isovolumetric Relaxation Time (IVRT)

The myocardial performance index (MPI) is a numerical value that can be used to assess ventricular function, and can be obtained by using cardiac time intervals. This numerical value is defined as the sum of the isovolumetric contraction time (IVCT) and the IVRT, divided by the ejection time(ET), and can be calculated for each ventricle individually (Figure 2). We calculated the RVMPI from the basal RV TDI using the formula: RV IVRT + RV IVCT / RV ejection time.

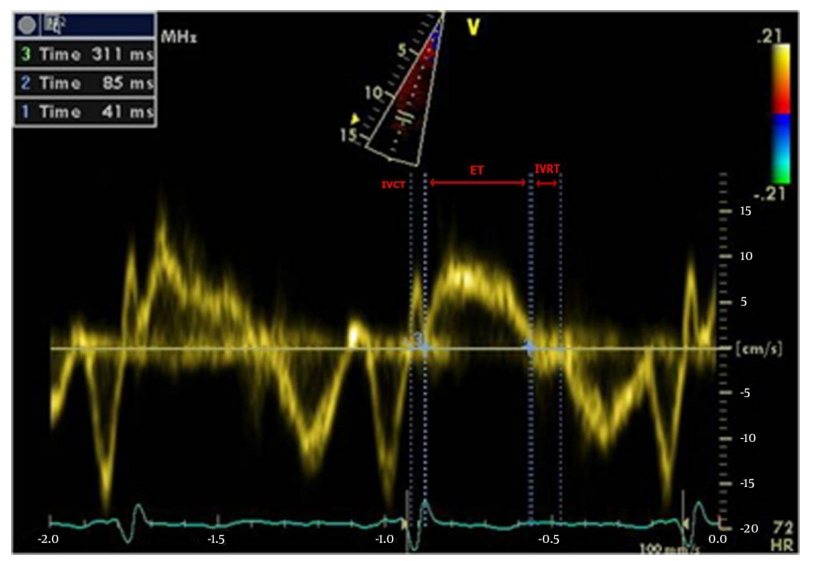

Figure 2. Isovolumetric Contraction Time (IVCT), Ejection Time (ET), Isovolumetric Relaxation Time (IVRT)

The TTP, a novel tissue Doppler variable, was measured as the time between the beginning of the IVCT to the peak of the systolic wave of the base of the RV free wall in the TDI (Figure 1). We used the $S$ velocity, tricuspid annular plane systolic excursion (TAPSE), and visual assessment to evaluate the RV systolic longitudinal function. The recordings 
were made at a sweep speed of $100 \mathrm{~mm} / \mathrm{sec}$, and an average of 3 cardiac cycles was used in each patient. All of the measurements were digitally recorded at end-expiration.

\subsection{Statistical Analysis}

The data were expressed as the mean \pm SD for the interval and count (percent) for the categorical variables. One sample Kolmogorov-Smirnov test was applied to investigate the fitness of the interval variables to normal distribution. For a better interpretation of the RHC results, a PAP of less than $25 \mathrm{mmHg}$ was considered to be normal. Comparisons between the study sub-groups were performed via the Student's t test (for the normally distributed interval data) and Pearson's chi square test (for the nominal data). The inter and intra-observer variability were determined by the paired sample t-test, and a P value $<0.05$ was considered to be statistically significant. The diagnostic accuracy of different echocardiographic indices compared to catheterization was determined by the area under the receiver operative characteristic (ROC) curve (AUC). Moreover, a multivariable analysis was performed to investigate the adjusted associations between certain important study variables and the PH via the logistic regression model. The statistical analyses were performed using SPSS 15.0 for Windows (SPSS Inc., Chicago, IL, USA).

\section{Results}

\subsection{Background Data}

Sixty patients, with a mean age of $44 \pm 14.4$ years (range: 14 - 67 years), and a female/male ratio of 42/18, participated in this study. Among them, 38 patients (63.3\%) had PAPs $\geq 25 \mathrm{mmHg}$, and were considered to have PH. The remaining 22 patients (36.7\%) had normal PAPs (non-PH). The patients' characteristics were compared between the two groups, and the results are presented in Table 1.

\section{2. "Time to Peak" and Pulmonary Hypertension}

As shown in Table 1, a significant difference was observed in the TTP between the PH and non-PH groups, with the $\mathrm{PH}$ group displaying a shorter mean TTP. This suggests that there was an inverse association between the $\mathrm{PH}$ and TTP. To investigate the accuracy of the TTP to diagnose PH, the AUC was applied. The AUC was $0.746 \pm 0.064(\mathrm{P}<$ 0.001) which suggested a fair diagnostic accuracy, while the cutoff point of $127 \mathrm{~ms}$ for the TTP had an acceptable sensitivity and specificity to diagnose PH (Figure 3).
Table 1. Background Characteristics of the Study Participants ${ }^{\mathrm{a}}$

\begin{tabular}{|c|c|c|c|}
\hline & $\operatorname{PH}(n=38)$ & Non-PH $(\mathbf{n}=22)$ & P Value \\
\hline Gender & & & 0.599 \\
\hline Male & 10 & 8 & \\
\hline Female & 28 & 14 & \\
\hline Age, $y$ & $46 \pm 13.3$ & $40 \pm 16.1$ & 0.120 \\
\hline BSA, $\mathrm{m}^{2}$ & $1.7 \pm 0.17$ & $1.7 \pm 0.16$ & 0.837 \\
\hline HR, beat/min & $76.1 \pm 9.4$ & $57.6 \pm 9.2$ & 0.001 \\
\hline Diagnosis & & & $<0.001$ \\
\hline VHD & $31(82)$ & $8(36)$ & \\
\hline CAD & $7(18)$ & $14(64)$ & \\
\hline IVRT, ms & & & 0.001 \\
\hline Ordinary & $73.7 \pm 11.7$ & $59.3 \pm 11.4$ & \\
\hline Corrected & $83.0 \pm 12$ & $57.4 \pm 9$ & \\
\hline RV MPI & $0.56 \pm 0.1$ & $0.4 \pm 0.1$ & 0.001 \\
\hline RV Size, cm & $3.4 \pm 0.66$ & $3.5 \pm 0.93$ & 0.508 \\
\hline TTP, ms & $127.2 \pm 24.3$ & $146.7 \pm 30.2$ & 0.008 \\
\hline $\mathrm{Sm}, \mathrm{cm} / \mathrm{sec}$ & $10.4 \pm 2.47$ & $12.4 \pm 2.04$ & 0.002 \\
\hline $\mathrm{Em}, \mathrm{cm} / \mathrm{sec}$ & $7.5 \pm 3.30$ & $6.8 \pm 4.28$ & 0.552 \\
\hline SVTI, cm & $17 \pm 5.80$ & $22.4 \pm 4.87$ & $<0.001$ \\
\hline TRG, mmHg & $44.3 \pm 18.18$ & $28.4 \pm 8.13$ & $<0.001$ \\
\hline \multicolumn{4}{|c|}{$\begin{array}{l}\text { Abbreviations: BSA, body surface area; HR, heart rate; VHD, valvular heart dis- } \\
\text { ease; CAD, coronary artery disease; IVRT, isovolumetric relaxation time; RV, } \\
\text { right ventricle; MPI, myocardial performance index; TTP, time to peak; VTI, } \\
\text { velocity-time integral; TRG, tricuspid regurgitation gradient; Sm, peak systolic } \\
\text { velocity; Em, early diastolic velocity; PH, pulmonary hypertension (mean PAP } \\
\geq 25 \mathrm{mmHg} \text { in right heart catheterization). } \\
\text { a Data presented as mean } \pm \text { standard deviation for interval and count (\%) for } \\
\text { categorical variables. }\end{array}$} \\
\hline
\end{tabular}

\subsection{RVIVRT and Pulmonary Hypertension}

When compared to the non-PH group, the PH group had a significantly longer IVRT $(\mathrm{P}<0.001)$ (Table 1$)$. After the correction for the heart rate, the differences in the IVRTs between the groups became greater. The AUC was applied for the later index, and the results showed excellent accuracy for the diagnosis of $\mathrm{PH}(\mathrm{AUC}=0.95 \pm 0.03$ ). According to these results, a heart-rate corrected IVRT $>68$ ms showed an optimal cutoff point, with a sensitivity of $94.7 \%$ and a specificity of $86.4 \%$ (Figure 4 ).

\subsection{Myocardial Performance Index and Pulmonary Hyperten- sion}

In the studied population, the MPI was significantly greater in the $\mathrm{PH}$ group, than in the non-PH group (0.56 \pm 0.1 vs. $0.4 \pm 0.1, \mathrm{P}=0.001$ ) (Table 1 ). The accuracy of this index for the diagnosis of PH was also assessed via the 


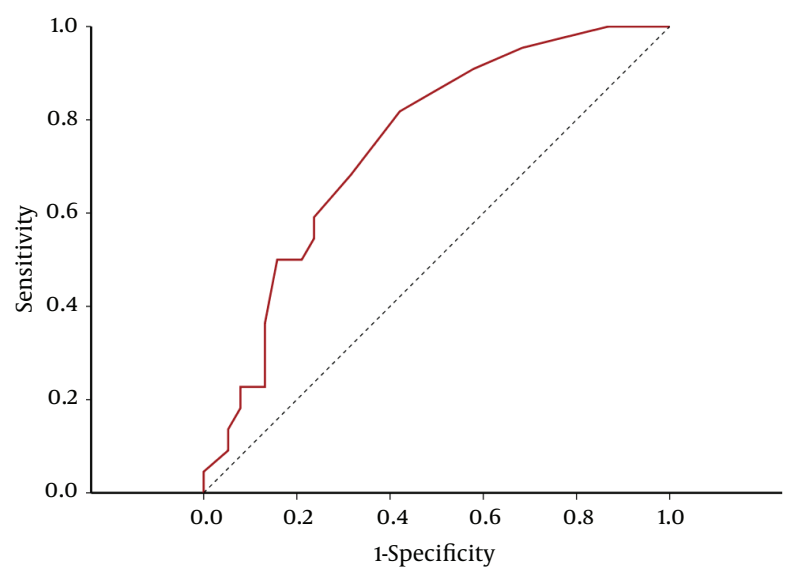

Figure 3. ROC curve illustrating a fair accuracy time duration, using the TTP for the diagnosis of pulmonary hypertension (PAP $\geq 25 \mathrm{mmHg}$ ). For the cutoff point of TTP $<127 \mathrm{~ms}$, the accuracy indices are presented above. All of the values are presented with their CI 95\% sensitivity: 0.68 (0.51 - 0.82), specificity: 0.68 (0.45 - 0.85), positive predictive value: $0.79(0.61-0.90)$, negative predictive value: $0.56(0.36-0.73)$, and area under the curve $=0.746 \pm 0.064$.

Figure 4. ROC Curve Illustrating the Excellent Accuracy of the IVRT for the Diagnosis of Pulmonary Hypertension (PAP $\geq 25 \mathrm{mmHg}$ )

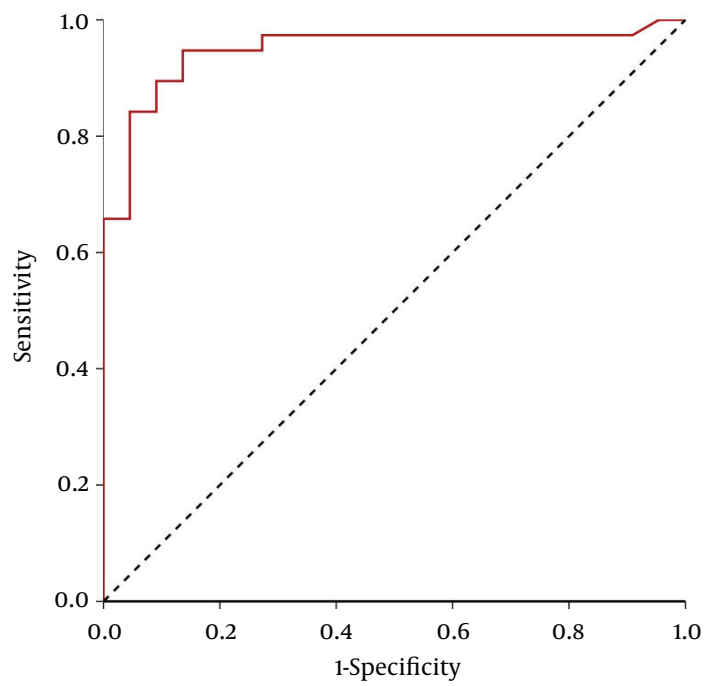

For the cut-off point of IVRT $>68 \mathrm{~ms}$, the accuracy indices are presented above. All of the values are presented with their CI $95 \%$ sensitivity: $94.7 \%(82.3 \%-99.4 \%)$, specificity: $86.4 \%$ (65.1\% - 97.1\%), positive predictive value: 92.3\% (79.1\% - 98.4\%), negative predictive value: $90.5 \%(69.6 \%-98.8 \%)$, and area under the curve: $0.95 \pm 0.03$.

ROC curve analysis. The computed AUC proposed good accuracy of the MPI for the diagnosis of PH (AUC $=0.86 \pm$ $0.06)$. We were interested in two cut-off points; one with an acceptable specificity, but better sensitivity, and the other with a good, equal sensitivity and specificity. The results are presented in Figure 5.
Figure 5. The ROC Curve Proposed Good Accuracy of the Myocardial Performance Index (MPI) for the Diagnosis of Pulmonary Hypertension (PAP $\geq 25 \mathrm{mmHg}$ )

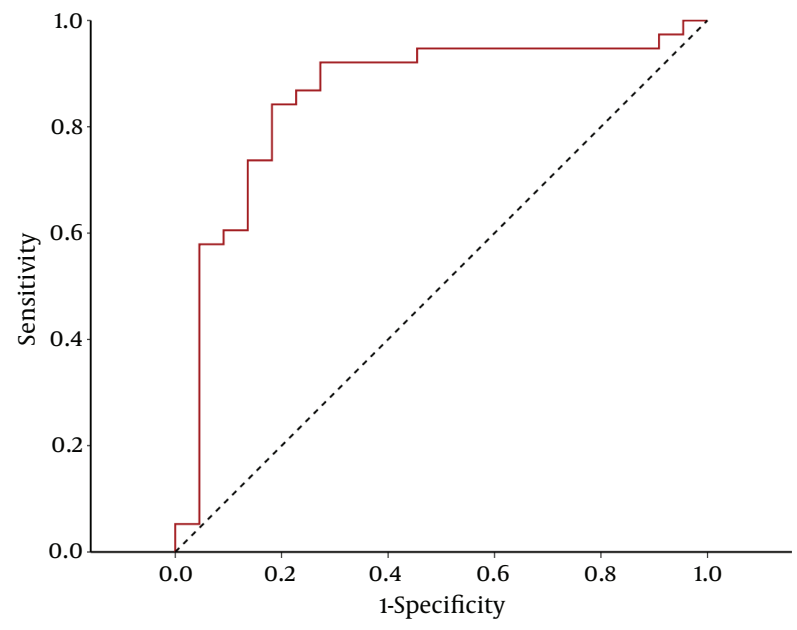

The accuracy indices are presented with their CI 95\% values for two different cut-off points. Area under the curve $=0.86 \pm 0.06$.

Figure 5 receiver operating characteristics (ROC) curve proposed a good accuracy of myocardial performance index (MPI) for the diagnosis of pulmonary hypertension. (PAP $\geq 25 \mathrm{mmHg}$ ). Accuracy indices were presented with their CI95\% for two different cut points. Area under the curve $=0.86 \pm 0.06$.

\subsection{Multivariable Analysis}

As shown in Table 1, the associations between other patients' characteristics and $\mathrm{PH}$ were investigated. The adjusted associations between the TTP and PH are presented in Table 2. In addition, the results of the logistic regression model showed that after adjusting for important predictors, no significant association was observed between the TTP and PH. However, a weak, direct association was found between the PH and IVRT. Other borderline direct associations were also observed between the PH, HR, and TRG (Table 2).

\subsection{Intra-Observer and Inter-Observer Variability}

The reproducibility of the IVRT was assessed in a subset of 30 patients ( 15 randomly selected participants from each group), and had good limits of interobserver and intraobserver variability. The mean differences between the two measurements made by the same examiner and two independent observers were $3 \pm 0.2 \mathrm{~ms}, \mathrm{P}=0.320$ and $5 \pm$ $0.5, \mathrm{P}=0.266$, respectively. 
Table 2. Binary Logistic Regression Model Showing the Adjusted Associations Between the Time to Peak and Pulmonary Hypertension

\begin{tabular}{lccc}
\hline & Coefficient \pm SE & P Value & Odds Ratio (CI 95\%) \\
\hline TTP, $<\mathbf{1 2 7} \mathbf{~ m s}$ & $-0.629 \pm 1.065$ & 0.554 & $0.533(0.066-4.295)$ \\
\hline HR, beat/min & $0.144 \pm 0.078$ & 0.064 & $1.155(0.992-1.346)$ \\
\hline Sm, cm/s & $-0.574 \pm 0.433$ & 0.185 & $0.564(0.241-1.316)$ \\
\hline SVTI, cm & $0.17 \pm 0.177$ & 0.338 & $1.185(0.838-1.676)$ \\
\hline TRG, $\mathbf{m m H g}$ & $0.164 \pm 0.089$ & 0.065 & $1.178(0.99-1.402)$ \\
\hline CAD & $-1.585 \pm 1.105$ & 0.152 & $0.205(0.023-1.788)$ \\
\hline AF Rhythm & $-1.127 \pm 1.554$ & 0.468 & $0.324(0.015-6.806)$ \\
\hline IVRT, ms & $0.105 \pm 0.045$ & 0.02 & $1.111(1.017-1.214)$ \\
\hline
\end{tabular}

Abbreviation: TTP, time to peak; HR, heart rate; Sm, peak systolic velocity; VTI, velocity-time integral; TRG, tricuspid regurgitation gradient; CAD, coronary artery disease; $\mathrm{AF}$, atrial fibrillation; IVRT, isovolumetric relaxation time.

\section{Discussion}

The PAP reflects both the left and right heart function, and thus, carries diagnostic, therapeutic, and prognostic values for patients with cardiac diseases $(1,2)$. The determination of the systolic PAP based on the TR is the most common echocardiographic method used. However, this method has various limitations and errors, such as requiring the presence of adequate Doppler signals and an optimal angle parallel to the jet. Although TR can be found in normal subjects, it is not always present. With the advent of technology, TDI has also been used in defining $\mathrm{PH}$ (9-11). TDI is superior to the blood flow Doppler, since it reflects the functional status of the myocardium directly, and is less subject to background noise and the loading condition (10). In several studies, the correlations between the RVIVRT from the TDI variables of the lateral tricuspid annulus and PAP have been evaluated (3, 7-13). The RV IVRT, that is, the time interval between the pulmonary valve closure (PVc) and tricuspid valve opening (TVo), has correlated well with the invasively measured SPAP in some previous studies (7-9).

Similar to certain studies, the current study also revealed a positive correlation between the RV IVRT and the mean PAP. This correlation was stronger for the corrected IVRT and mean PAP. In 2013, Zimbarra Cabrita et al. (13) studied the correlation between the corrective IVRT and systolic PAP measured via echocardiography, in which a corrected IVRT $>75 \mathrm{~ms}$ showed a strong correlation with $\mathrm{PH}$. In the present study, we measured the mean PAP via right heart catheterization, which is the standard for the diagnosis of $\mathrm{PH}$. We also found a strong correlation between the corrected IVRT and the mean PAP. Moreover, the effects of the $\mathrm{RV}$ systolic function were also investigated in this correla- tion. This study only showed a strong relationship $\mathrm{n}$ those patients with no significant RV dysfunction. In addition, a weak correlation was found between the corrected RV IVRT and mean PAP in the patients with significant RV dysfunction. It appears that RV dysfunction reduces the rate of the RV pressure drop during relaxation, increasing the diastolic PAP and lengthening the RV IVRT. Therefore, we suggest that caution should be taken when using the corrected RV IVRT to predict the PH in those patients with significant RV dysfunction.

The MPI, which is also known as the Tei index, is a Doppler-derived time interval index that combines both the systolic and diastolic cardiac performances. Furthermore, the Tei index has been shown to have a strong prognostic value in certain cardiac diseases, such as PH (14). Because of the potent systolic parameters that contribute to the Tei index, such as the IVCT and ET, this index reliably detects the current alterations of the ventricular systolic function. Therefore, it maintains a strong inverse relationship with the ejection fraction: the higher the value of the index, the lower the ejection fraction. Furthermore, increased RV afterload, which is associated with the $\mathrm{PH}$, has a negative impact on the RV systolic function.

Hilde et al. (15) showed a significant correlation between the mean PAP and RV MPI, and suggested that the RV MPI value is higher, even at slight elevations in the mean PAP. Our study also revealed a positive correlation between the RV MPI and PH. Moreover, we found that a cut-off value of an RV MPI > 0.42 can be used to predict PH. This finding highlights the value of the RV MPI in predicting PH, in addition to its prognostic significance in these patients.

In the McLean et al. study (16), in which the systolic PAP was estimated from the TR signal, the TTP/RV diameter was suggested as a useful parameter to predict PH. LopezCandales et al. (17) studied the effects of PH on the isovolumetric contraction (IVC) and systolic (S) signal's TDIs of both ventricles, and showed that the PH markedly shortened the time interval between the IVC and the tricuspid annular systolic signal, when compared to the individuals without $\mathrm{PH}(\mathrm{P}<0.0001)$. In our study, a significantly inverse correlation was found between the TTP and the mean PAP measured by catheterization.

These results show that the RV TDI can be used to predict the elevated mean PAP. Given its rapid acquisition times, reproducibility, and ease of addition to standard transthoracic echocardiographic protocols, the RV TDI is an important additional modality in comprehensive echocardiographic studies (18).

\subsection{Limitations}

The results of this study should be interpreted in light of certain limitations, since we focused on hemodynam- 
ically stable patients with chronic $\mathrm{PH}$, and it is unclear whether our results would also apply to decompensated or acute PH cases. Additionally, a graded estimation of the PAP was not considered, which limited our study to a dichotomous PAP level.

\subsection{Conclusion}

The present study, in agreement with certain other studies, showed a strong positive correlation between the corrected RV IVRT, as measured by the TDI and mean PAP. However, we found this correlation to be weak in the presence of significant RV systolic dysfunction. In fact, the RV dysfunction perturbs the evaluation of the PAP using the RV IVRT. Moreover, the RV MPI was found to have a positive correlation with the mean PAP, and can be used for the prediction of $\mathrm{PH}$, in addition to its prognostic value in these cases.

In the present study, a good inverse correlation was observed between the TTP and the mean PAP. It could be concluded that this new tissue Doppler index could be considered as a predictive factor for PH. We suggest the use of the novel parameter "time to peak" in addition to the previous standard echocardiographic parameters in those patients suspicious for $\mathrm{PH}$.

\section{Acknowledgments}

The authors wish to thank Dr. Hojjat Ghaderi and Dr. Toktam Moghiman for their helpful assistance in the editing of this paper. They especially thank the echocardiography department and catheterization laboratory at the Rajaie cardiovascular medical and research center for supporting this study.

\section{Footnote}

Authors' Contributions: Mozhgan Parsaee and Fereshteh Ghaderi realized the study concept and design; Azin Alizadehasl contributed in the analysis and interpretation of the data; Fereshteh Ghaderi wrote and submitted the manuscript; Hooman Bakhshandeh performed data analysis, contribute in the writing and revised the manuscript. Each author has read and sufficiently contributed to this manuscript.

\section{References}

1. Eduardo B, Antonello DA, Michele DA. Echocardigraphy in PAH: from diagnosis to prognosis. J Am soc Echocardiogr. 2013;26:1-14.

2. Hoeper MM, Bogaard HJ, Condliffe R, Frantz R, Khanna D, Kurzyna $\mathrm{M}$, et al. Definitions and diagnosis of pulmonary hypertension. J Am Coll Cardiol. 2013;62(25 Suppl):D42-50. doi: 10.1016/j.jacc.2013.10.032. [PubMed: 24355641].
3. Milan A, Magnino C, Veglio F. Echocardiographic indexes for the noninvasive evaluation of pulmonary hemodynamics. J Am Soc Echocardiogr. 2010;23(3):225-39. doi: 10.1016/j.echo.2010.01.003. [PubMed: 20206827] quiz 332-4.

4. Steckelberg RC, Tseng AS, Nishimura R, Ommen S, Sorajja P. Derivation of mean pulmonary artery pressure from noninvasive parameters. $J$ Am Soc Echocardiogr. 2013;26(5):464-8. doi:10.1016/j.echo.2013.01.006. [PubMed: 23411365].

5. Howard LS, Grapsa J, Dawson D, Bellamy M, Chambers JB, Masani ND, et al. Echocardiographic assessment of pulmonary hypertension: standard operating procedure.Eur Respir Rev. 2012;21(125):239-48. doi: 10.1183/09059180.00003912. [PubMed: 22941889].

6. McLaughlin VV, Archer SL, Badesch DB, Barst RJ, Farber HW, Lindner JR, et al. ACCF/AHA 2009 expert consensus document on pulmonary hypertension a report of the American College of Cardiology Foundation Task Force on Expert Consensus Documents and the American Heart Association developed in collaboration with the American College of Chest Physicians; American Thoracic Society, Inc.; and the Pulmonary Hypertension Association. J Am Coll Cardiol. 2009;53(17):1573619. doi: 10.1016/j.jacc.2009.01.004. [PubMed: 19389575].

7. Brechot N, Gambotti L, Lafitte S, Roudaut R. Usefulness of right ventricular isovolumic relaxation time in predicting systolic pulmonary artery pressure. Eur J Echocardiogr. 2008;9(4):547-54. doi: 10.1093/ejechocard/jen121. [PubMed: 18490309].

8. Ernande L, Cottin V, Leroux PY, Girerd N, Huez S, Mulliez A, et al. Right isovolumic contraction velocity predicts survival in pulmonary hypertension. J Am Soc Echocardiogr. 2013;26(3):297-306. doi: 10.1016/j.echo.2012.11.011. [PubMed: 23265440].

9. Dambrauskaite V, Delcroix M, Claus P, Herbots L, Palecek T, D’Hooge J, et al. The evaluation of pulmonary hypertension using right ventricular myocardial isovolumic relaxation time. J Am Soc Echocardiogr. 2005;18(11):1113-20. doi: 10.1016/j.echo.2005.07.019. [PubMed: 16275518].

10. Ruan Q, Nagueh SF. Clinical application of tissue Doppler imaging in patients with idiopathic pulmonary hypertension. Chest. 2007;131(2):395-401. doi:10.1378/chest.06-1556. [PubMed: 17296639].

11. McQuillan BM, Picard MH, Leavitt M, Weyman AE. Clinical correlates and reference intervals for pulmonary artery systolic pressure among echocardiographically normal subjects. Circulation. 2001;104(23):2797-802. [PubMed: 11733397].

12. Grapsa J, Dawson D, Nihoyannopoulos P. Assessment of right ventricular structure and function in pulmonary hypertension.J Cardiovasc Ultrasound. 2011;19(3):115-25. doi: 10.4250/jcu.2011.19.3.115. [PubMed: 22073320].

13. Zimbarra Cabrita I, Ruisanchez C, Grapsa J, Dawson D, North B, Pinto FJ, et al. Validation of the isovolumetric relaxation time for the estimation of pulmonary systolic arterial blood pressure in chronic pulmonary hypertension. Eur Heart J Cardiovasc Imaging. 2013;14(1):51-5. doi: 10.1093/ehjci/jes093. [PubMed: 22588209].

14. Horton KD, Meece RW, Hill JC. Assessment of the right ventricle by echocardiography: a primer for cardiac sonographers. $J$ Am Soc Echocardiogr. 2009;22(7):776-92. doi: 10.1016/j.echo.2009.04.027. [PubMed: 19560657] quiz 861-2.

15. Hilde JM, Skjorten I, Grotta OJ, Hansteen V, Melsom MN, Hisdal J, et al. Right ventricular dysfunction and remodeling in chronic obstructive pulmonary disease without pulmonary hypertension. J Am Coll Cardiol. 2013;62(12):1103-11. doi: 10.1016/j.jacc.2013.04.091. [PubMed: 23831444].

16. McLean AS, Ting I, Huang SJ, Wesley S. The use of the right ventricular diameter and tricuspid annular tissue Doppler velocity parameter to predict the presence of pulmonary hypertension. Eur J Echocardiogr. 2007;8(2):128-36. doi: 10.1016/j.euje.2006.02.005. [PubMed:16672193]. 
17. Lopez-Candales A, Bazaz R, Edelman K, Gulyasy B. Altered early left ventricular diastolic wall velocities in pulmonary hypertension: a tissue Doppler study. Echocardiography. 2009;26(10):1159-66. doi: 10.1111/j.1540-8175.2009.00944.x. [PubMed: 19765074].

18. Bech-Hanssen O, Karason K, Rundqvist B, Bollano E, Lindgren F, Se- limovic N. Can pulmonary hypertension and increased pulmonary vascular resistance be ruled in and ruled out by echocardiography?.J Am Soc Echocardiogr. 2013;26(5):469-78. doi:10.1016/j.echo.2013.02.011. [PubMed: 23510537]. 